\title{
Color Image Compression using SPIHT Algorithm
}

\author{
Sadashivappa ${ }^{1}$ \\ Mahesh Jayakar ${ }^{1 . A}$ \\ 1. Professor, \\ 1. a. Junior Research Fellow, \\ Dept. of Telecommunication \\ R.V College of Engineering, \\ Bangalore-59, India
}

\author{
K.V.S Anand Babu ${ }^{2}$ \\ 2. Principal \\ C.M.R Institute of Technology \\ Bangalore-37 \\ INDIA.
}

\author{
Dr. Srinivas $\mathrm{K}^{3}$ \\ 3. DBSD, NRSC \\ Hyderabad-5600625 \\ INDIA
}

\begin{abstract}
In recent years there has been an astronomical increase in the usage of computers for a variety of tasks. With the advent of digital cameras, one of the most common uses has been the storage, manipulation, and transfer of digital images. The files that comprise these images, however, can be quite large and can quickly take up precious memory space on the computer's hard drive. In multimedia application, most of the images are in color. And color images contain lot of data redundancy and require a large amount of storage space. In this work, we are presenting the performance of different wavelets using SPIHT[1] algorithm for compressing color image. In this R, G and B component of color image are converted to $\mathrm{YCbCr}$ before wavelet transform is applied. $\mathrm{Y}$ is luminance component; $\mathrm{Cb}$ and $\mathrm{Cr}$ are chrominance components of the image. Lena color image is taken for analysis purpose. Image is compressed for different bits per pixel by changing level of wavelet decomposition. Matlab software is used for simulation. Results are analyzed using PSNR and HVS property. Graphs are plotted to show the variation of PSNR for different bits per pixel and level of wavelet decomposition.
\end{abstract}

\section{keywords:}

SPIHT, Color Image, Wavelet, luminance, chrominance.

\section{INTRODUCTION}

In digital true color image, each color component that is R, G, B components, each contains 8 bit data[3]. Also color image contains lots of redundancy which will make it difficult to store and transmit. However, RGB [3] model is not suited for image processing purpose. For compression, a luminance-chrominance representation is considered due to superior to the RGB representation. Therefore, RGB images are transformed to one of the luminance-chrominance models, performing the compression process, and then transform back to RGB model because displays are most often provided output image with direct RGB model. The luminance component represents the intensity of the image and looks like a gray scale version. The chrominance components represent the color information in the image. The rest of the paper is organized as follows: Wavelet Transformation of Image is described in section II. SPIHT[1] algorithm is explained in section III. Modeling and results is given in section IV. Conclusion and Future work is explained in section V.

\section{WAVELET TRANSFORMATION OF IMAGES}

Wavelets[7] are mathematical functions that decompose data into different frequency components, and then study each component with a resolution matched to its scale. They have advantages over traditional Fourier methods[4] in analyzing physical situations where the signal contains discontinuities and sharp spikes. Wavelets were developed independently in the fields of mathematics, quantum physics, electrical engineering, and seismic geology. Interchanges between these fields during the last ten years have led to many new wavelet applications such as image compression, turbulence, human vision, radar, and earthquake prediction.

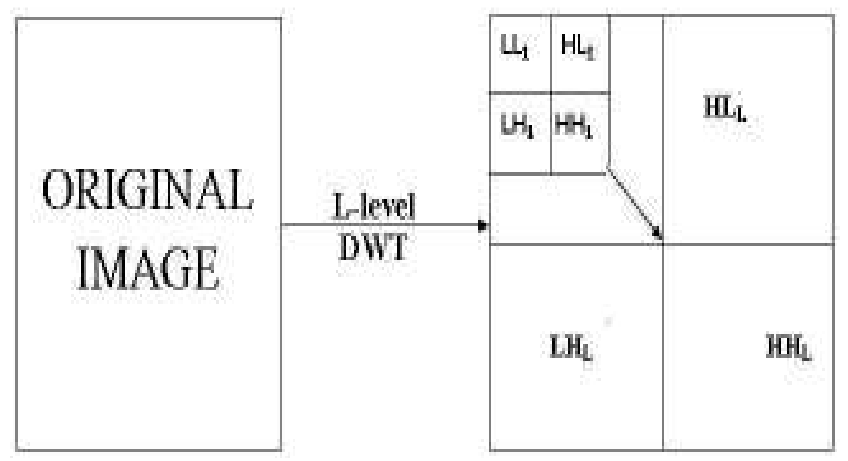

Figure 1: Wavelet Transform

The wavelet transformation[7] is a mathematical tool for decomposition. The wavelet transform is identical to a hierarchical sub band filtering system[3], where the sub bands are logarithmically spaced in frequency. The basic idea of the DWT for a two-dimensional image is described as follows. An image is first decomposed into four parts based on frequency sub bands, by critically sub sampling horizontal and vertical channels using sub band filters and named as Low-Low (LL), Low-High (LH), HighLow (HL), and High- High (HH) sub bands as shown in figure 1. To obtain the next coarser scaled wavelet coefficients, the sub band LL is further decomposed and critically sub sampled. This process is repeated several times, which is determined by the application at hand. The block diagram of this process is shown in figure 1. Each level has various bands information such as lowlow, low-high, high-low, and high-high frequency bands. Furthermore, from these DWT coefficients, the original image can be reconstructed. This reconstruction process is called the inverse DWT (IDWT). If $\mathrm{C}[\mathrm{m}, \mathrm{n}]$ represents an image, the DWT and 
IDWT for $\mathrm{C}[\mathrm{m}, \mathrm{n}]$ can similarly be defined by implementing the DWT and IDWT on each dimension and separately.

\section{SPIHT ALGORITHM}

The SPIHT[1] image coding algorithm was developed in 1996 by Said and Pearlman and is another more efficient implementation of the embedded zerotree wavelet (EZW)[2][8] algorithm by Shapiro. After the wavelet transform is applied to an image, the main algorithm works by partitioning the wavelet decomposed image into significant and insignificant partitions based on the following function:

$$
S_{n}(T)=\left\{\begin{array}{l}
1, \max _{(i, j) \in T}\left\{\left|c_{i, j}\right|\right\} \geq 2^{n} \\
0, \text { Otherwise }
\end{array}\right\}------\mathrm{Eq} 1
$$

Where $\operatorname{Sn}(T)$, is the significance of a set of co-ordinates T, and $c i, j$ is the coefficient value at co-ordinate $(i, j)$. There are two passes in the algorithm - the sorting pass and the refinement pass. The sorting pass is performed on the list of insignificant sets (LIS), list of insignificant pixels (LIP) and the list of significant pixels (LSP). The LIP and LSP consist of nodes that contain single pixels, while the LIS contains nodes that have descendants. The maximum number of bits required to represent the largest coefficient in the spatial orientation tree is obtained and designated as $\mathrm{n}_{\max }$, which is

$$
n_{\text {max }}=\left[\log _{2}\left(\max _{\mathrm{i}, \mathrm{j}}\left\{\left|c_{i, j}\right|\right\}\right)\right] \ldots \ldots . \mathrm{Eq} 2 .
$$

During the sorting pass, those co-ordinates of the pixels which remain in the LIP are tested for significance by using eqn. 2 . The result, $S,(T)$, is sent to the output. Those that are significant will be transferred to the LSP as well as having their sign bit output. Sets in the LIS (which consists of nodes with descendants will also have their significance tested and, if found to be significant, will be removed and partitioned into subsets. Subsets with a single coefficient and found to be significant will be added to the LSP, or else they will be added to the LIP. During the refinement pass, the nth most significant bit of the coefficients in the LSP is output. The value of $n$ is decreased by 1 and the sorting and refinement passes are repeated. This continues until either the desired rate is reached or $n=0$, and all the nodes in the LSP have all their bits output. The latter case will result in almost perfect reconstruction as all the coefficients are processed completely. The bit rate can be controlled precisely in the SPIHT[1] algorithm because the output produced is in single bits and the algorithm can be terminated at any time. The decoding process follows the encoding exactly and is almost symmetrical in terms of processing time.

\section{MODELING AND RESULTS}

Color image compression[3][4] is very important in today's communication era because most of the images are in color. Color images take more space for storage. Also without compression it may take long time for transferring images through internet. Figure 4 shows the model used for compressing color images. Matlab software is used for simulating this work. In our analysis we have used lena true color image (RGB 24 bit). Image is converted to $\mathrm{YCbCr}$ format. $\mathbf{Y C b C r}$ or $\mathbf{Y}^{\prime} \mathbf{C b C r}$, sometimes written $\mathrm{YC}_{\mathrm{B}} \mathrm{C}_{\mathrm{R}}$ or $\mathrm{Y}^{\prime} \mathrm{C}_{\mathrm{B}} \mathrm{C}_{\mathrm{R}}$, is a family of color spaces used as a part of the color image pipeline in video and digital photography systems. $Y^{\prime}$ is the luma component and $C_{B}$ and $C_{R}$ are the bluedifference and red-difference chroma components. $\mathrm{Y}^{\prime}$ (with prime) is distinguished from $\mathrm{Y}$ which is luminance, meaning that light intensity is non-linearly encoded using gamma correction. Figure 3 shows $\mathrm{YCbCr}$ image. After converting wavelet analysis is done for $Y, C_{B}, C_{R}$. Then the data is compressed using SPIHT[1] algorithm. Lena image shown below is used for analysis. For calculating PSNR only Y (Luminance) component of original and reconstructed image is used. Lena image shown in figure 2 is used for our analysis. Following are the result for different wavelets.

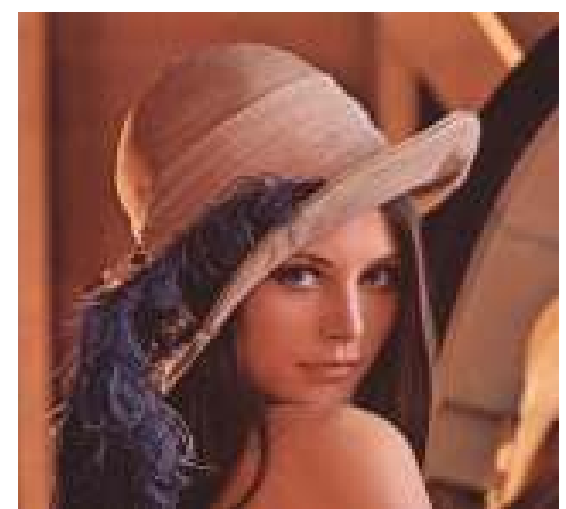

Figure 2: RGB Lena Image

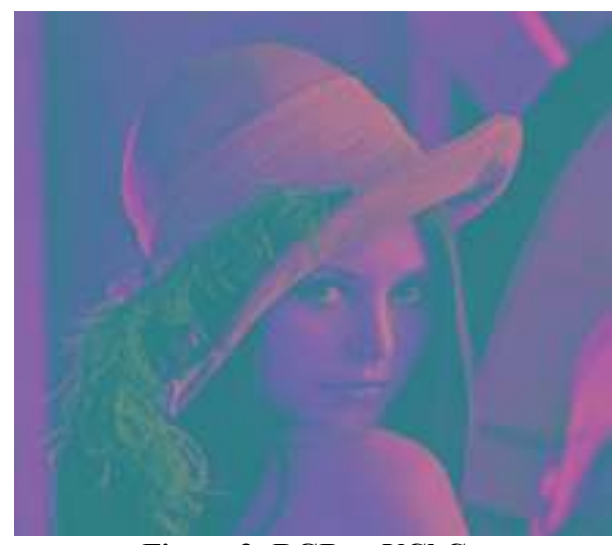

Figure 3: RGB to YCbCr 

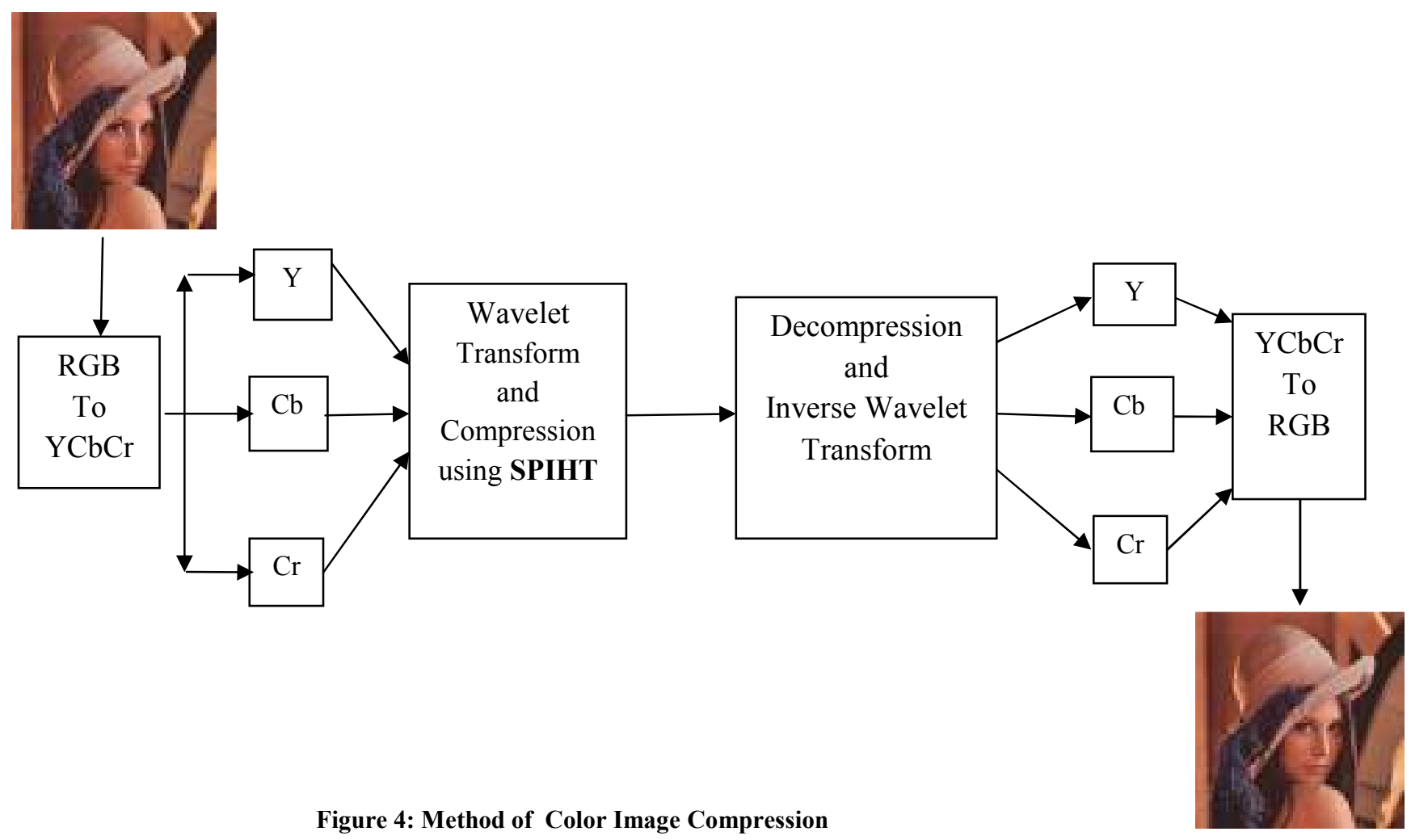

Figure 4: Method of Color Image Compression

\section{- bior 1.5}

Table 1.1 shows PSNR values for wavelet bior1.5 for different level of decomposition. Bits per Pixel are kept at 1bpp for our analysis and level of decomposition is kept fixed to 3. Figure 5 shows the PSNR response plotted for the values given in table 1.1. Figure 6 shows the reconstructed image for bior 1.5 wavelet.

Table1.1: PSNR for different level of wavelet decomposition for bior1.5

\begin{tabular}{|l|l|l|l|l|l|}
\hline bpp & 0.0625 & 0.125 & 0.25 & 0.5 & 1 \\
\hline Level & \multicolumn{5}{|c|}{ PSNR } \\
\hline 1 & 44.417 & 39.20 & 34.31 & 31.72 & 31.32 \\
\hline 2 & 34.276 & 31.77 & 31.36 & 30.24 & 30.01 \\
\hline 3 & 31.355 & 30.21 & 29.81 & 32.67 & 36.53 \\
\hline
\end{tabular}

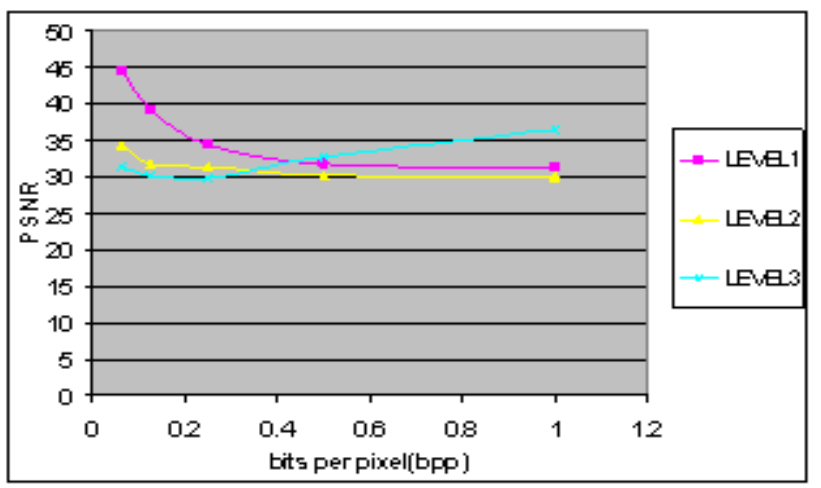

Figure 5 PSNR response for bior1.5

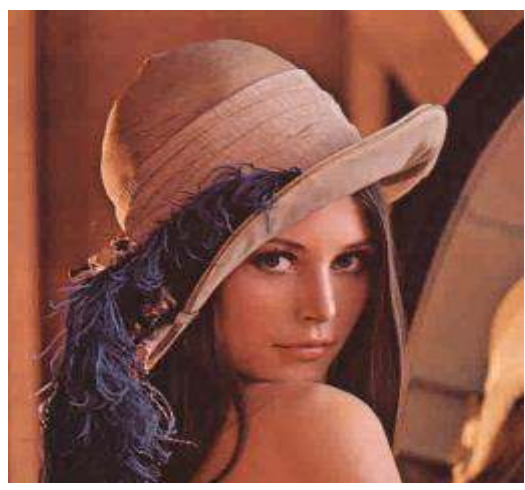

Figure 6: Reconstructed Image for bior1.5

\section{- bior 2.8}

Table 1.2 shows PSNR values for wavelet bior2.8 for different level of decomposition. Bits per Pixel are kept at $1 \mathrm{bpp}$ for our analysis and level of decomposition is kept fixed to 3.. Figure 7 shows the PSNR response plotted for the values given in table 1.2. Figure 8 shows the reconstructed image for bior 2.8 wavelet.

Table 1.2 PSNR for different level of wavelet decomposition for bior2.8

\begin{tabular}{|l|l|l|l|l|l|}
\hline bpp & 0.0625 & 0.125 & 0.25 & 0.5 & 1 \\
\hline level & \multicolumn{5}{|c|}{ PSNR } \\
\hline 1 & 45.24 & 39.78 & 34.88 & 32.23 & 31.80 \\
\hline 2 & 34.96 & 32.43 & 31.98 & 30.60 & 30.38 \\
\hline 3 & 32.36 & 30.90 & 30.23 & 33.76 & 38.37 \\
\hline
\end{tabular}




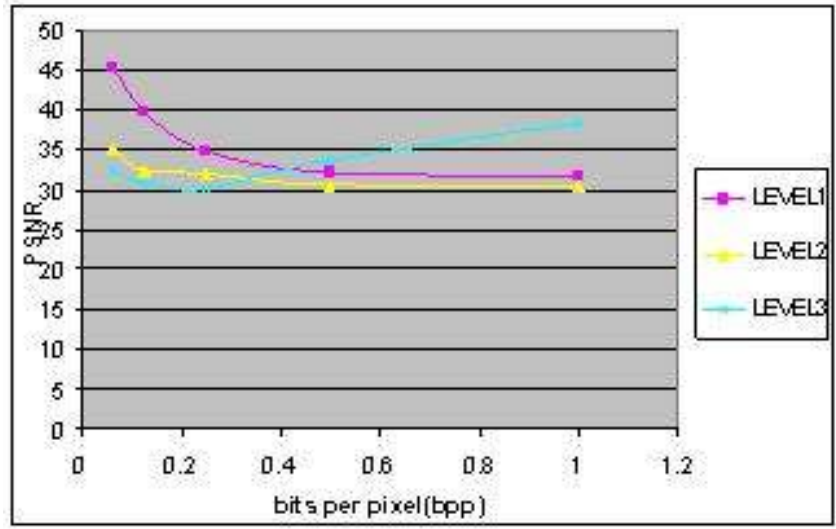

Figure 7 PSNR response for bior2.8

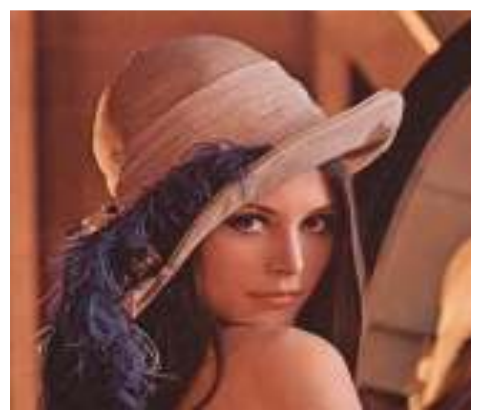

Figure 8 Reconstructed Image for bior 2.8

\section{- bior 3.7}

Table 1.3 shows PSNR values for wavelet bior3.7 for different level of decomposition. Bits per Pixel are kept at $1 \mathrm{bpp}$ for our analysis and level of decomposition is kept fixed to 3. Figure 9 shows the PSNR response plotted for the values given in table 1.3. Figure 10 shows the reconstructed image for bior 3.7 wavelet.

Table 1.3 PSNR for different level of wavelet decomposition for bior3.7

\begin{tabular}{|l|l|l|l|l|l|}
\hline bpp & 0.0625 & 0.125 & 0.25 & 0.5 & 1 \\
\hline Level & \multicolumn{5}{|c|}{ PSNR } \\
\hline 1 & 45.41 & 40.07 & 35.13 & 32.38 & 31.93 \\
\hline 2 & 62.00 & 62.00 & 62.00 & 35.50 & 29.73 \\
\hline 3 & 62.63 & 35.53 & 29.70 & 32.44 & 37.35 \\
\hline
\end{tabular}

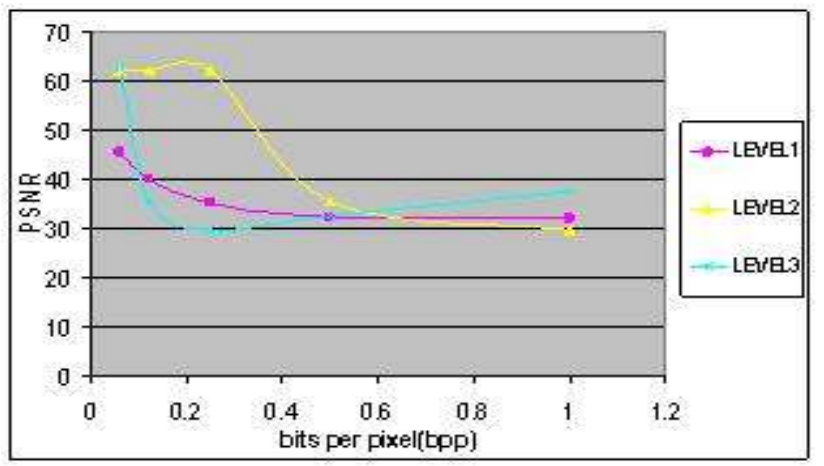

Figure 9 PSNR response of bior3.7

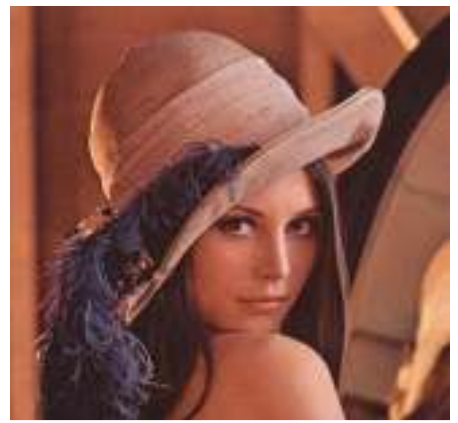

Figure 10 Reconstructed Image for bior3.7

\section{- bior 5.5}

Table 1.4 shows PSNR values for wavelet bior5.5 for different level of decomposition. Bits per Pixel are kept at $1 \mathrm{bpp}$ for our analysis and level of decomposition is kept fixed to 3 . Figure 11 shows the PSNR response plotted for the values given in table 1.4. Figure 12 shows the reconstructed image for bior 5.5 wavelet.

Table 1.4 PSNR for different level of wavelet decomposition for bior5.5

\begin{tabular}{|l|l|l|l|l|l|}
\hline bpp & 0.0625 & 0.125 & 0.25 & 0.5 & 1 \\
\hline Level & \multicolumn{5}{|c|}{ PSNR } \\
\hline 1 & 44.99 & 39.42 & 34.52 & 31.99 & 31.59 \\
\hline 2 & 34.51 & 32.07 & 31.69 & 30.35 & 29.76 \\
\hline 3 & 32.01 & 30.38 & 29.31 & 31.45 & 37.71 \\
\hline
\end{tabular}

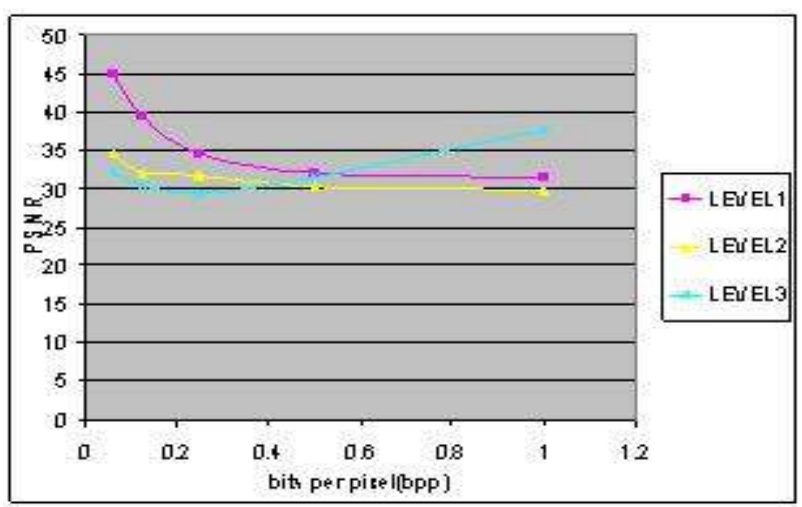

Figure 11 PSNR response for bior5.5

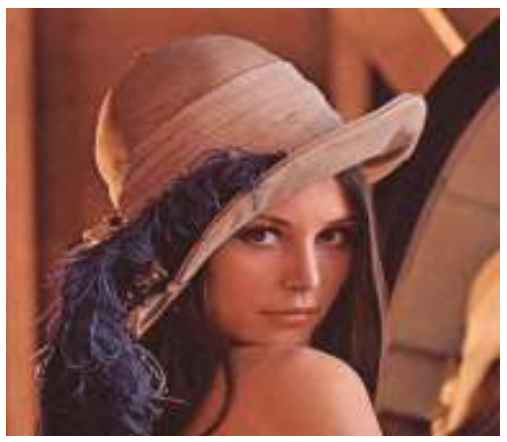

Figure 12 Reconstructed image for bior5.5 


\section{- coif2}

Table 1.5 shows PSNR values for wavelet coif2 for different level of decomposition. Bits per Pixel are kept at $1 \mathrm{bpp}$ for our analysis and level of decomposition is kept fixed to 3. Figure 13 shows the PSNR response plotted for the values given in table 1.5. Figure 14 shows the reconstructed image for coif 2 wavelet.

Table 1.5 PSNR for different level of wavelet decomposition for coif2

\begin{tabular}{|l|l|l|l|l|l|}
\hline bpp & 0.0625 & 0.125 & 0.25 & 0.5 & 1 \\
\hline Level & \multicolumn{5}{|c|}{ PSNR } \\
\hline 1 & 45.04 & 39.57 & 34.67 & 32.09 & 31.67 \\
\hline 2 & 34.55 & 32.06 & 31.64 & 30.41 & 30.01 \\
\hline 3 & 31.99 & 30.63 & 29.89 & 33.03 & 38.32 \\
\hline
\end{tabular}

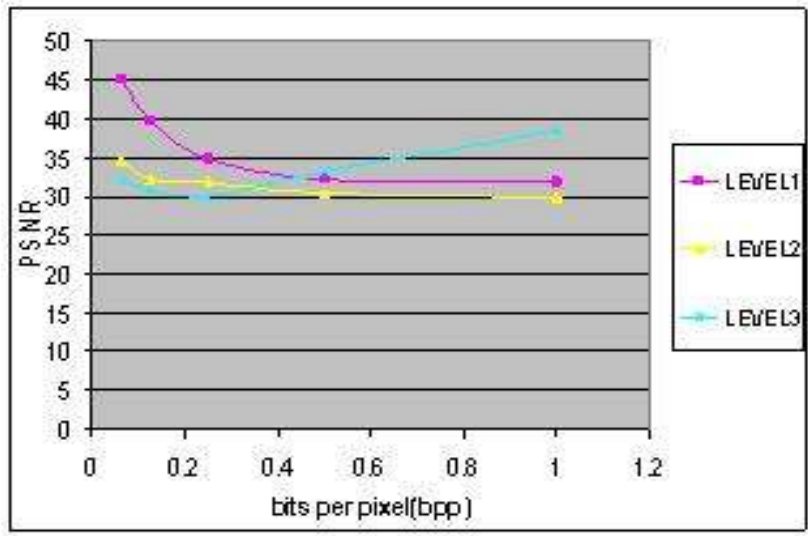

Figure 13 PSNR response of coif2

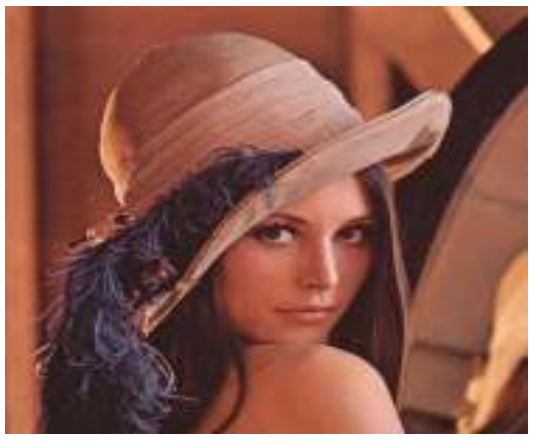

Figure 14 Reconstructed Image for coif2

\section{- coif4}

Table 1.6 shows PSNR values for wavelet coif4 for different level of decomposition. Bits per Pixel are kept at 1bpp for our analysis and level of decomposition is kept fixed to 3 . Figure 15 shows the PSNR response plotted for the values given in table 1.6. Figure 16 shows the reconstructed image for coif4 wavelet.

Table 1.6 PSNR for different level of wavelet decomposition for coif 4

\begin{tabular}{|l|l|l|l|l|l|}
\hline bpp & 0.0625 & 0.125 & 0.25 & 0.5 & 1 \\
\hline
\end{tabular}

\begin{tabular}{|l|l|l|l|l|l|}
\hline Level & \multicolumn{5}{|c|}{ PSNR } \\
\hline 1 & 45.01 & 39.64 & 34.67 & 32.10 & 31.67 \\
\hline 2 & 34.61 & 32.15 & 31.72 & 30.45 & 30.03 \\
\hline 3 & 31.91 & 30.62 & 29.74 & 32.94 & 38.38 \\
\hline
\end{tabular}

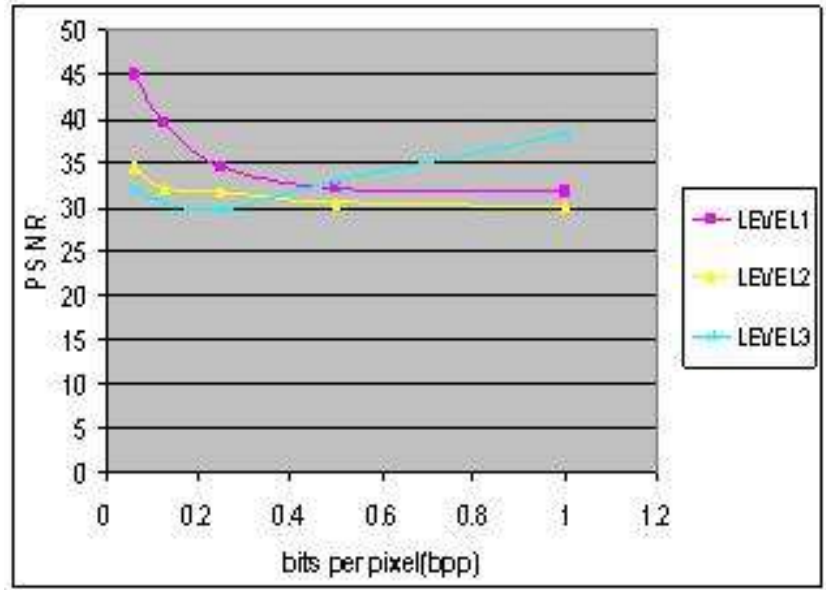

Figure 15 PSNR response of coif4

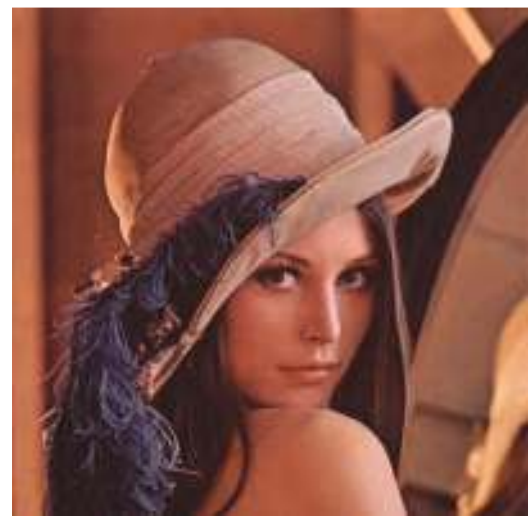

Figure 16 Reconstructed image for coif 4

- $\quad$ db3

Table 1.7 shows PSNR values for wavelet db3 for different level of decomposition. Bits per Pixel are kept at $1 \mathrm{bpp}$ for our analysis and level of decomposition is kept fixed to 3. Figure 17 shows the PSNR response plotted for the values given in table 1.7. Figure 18 shows the reconstructed image for $\mathrm{db} 3$ wavelet.

Table 1.7: PSNR for different level of wavelet decomposition for $\mathrm{db3}$

\begin{tabular}{|c|c|c|c|c|c|}
\hline bpp & 0.0625 & 0.125 & 0.25 & 0.5 & 1 \\
\hline Level & \multicolumn{5}{|c|}{ PSNR } \\
\hline 1 & 44.99 & 39.56 & 32.07 & 34.63 & 31.64 \\
\hline 2 & 34.61 & 32.16 & 30.47 & 31.73 & 30.06 \\
\hline 3 & 32.05 & 30.66 & 32.91 & 29.84 & 38.20 \\
\hline
\end{tabular}




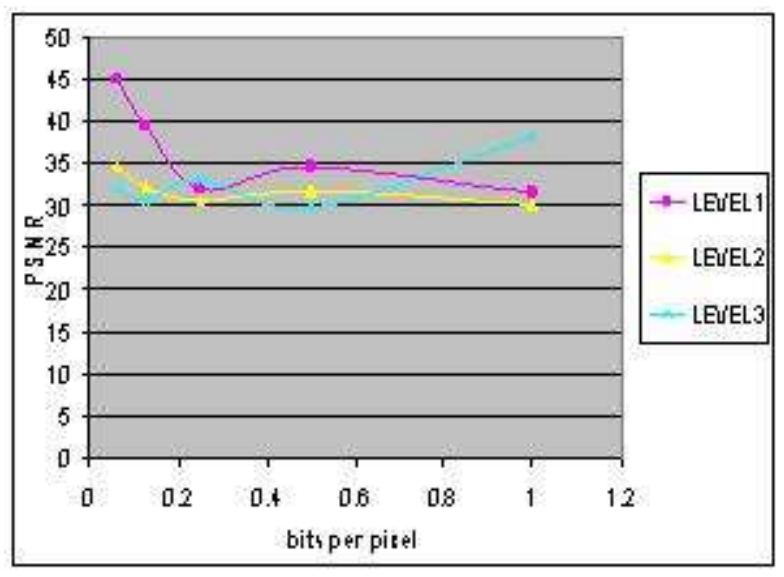

Figure 17: PSNR response of $\mathrm{db3}$

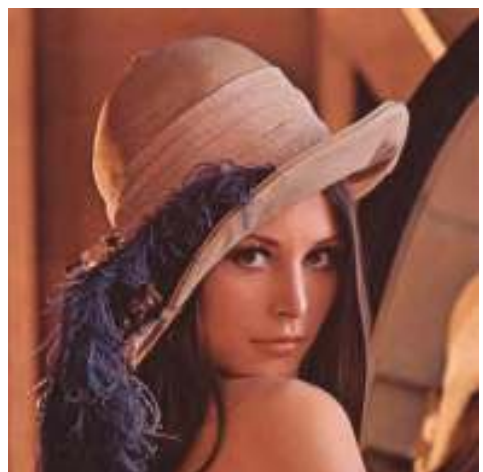

Figure 18:Reconstructed image for $\mathrm{db3}$

\section{- $\mathbf{d b 6}$}

Table 1.8 shows PSNR values for wavelet db6 for different level of decomposition. Bits per Pixel are kept at $1 \mathrm{bpp}$ for our analysis and level of decomposition is kept fixed to 3. Figure 19 shows the PSNR response plotted for the values given in table 1.8. Figure 20 shows the reconstructed image for db6 wavelet.

Table 1.8 PSNR for different level of wavelet decomposition for $\mathbf{d b 6}$

\begin{tabular}{|l|l|l|l|l|l|}
\hline bpp & 0.0625 & 0.125 & 0.25 & 0.5 & 1 \\
\hline Level & \multicolumn{5}{|c|}{ PSNR } \\
\hline 1 & 45.22 & 39.81 & 34.71 & 32.10 & 31.68 \\
\hline 2 & 34.69 & 32.25 & 31.80 & 30.52 & 30.00 \\
\hline 3 & 32.11 & 30.73 & 29.70 & 32.89 & 38.24 \\
\hline
\end{tabular}

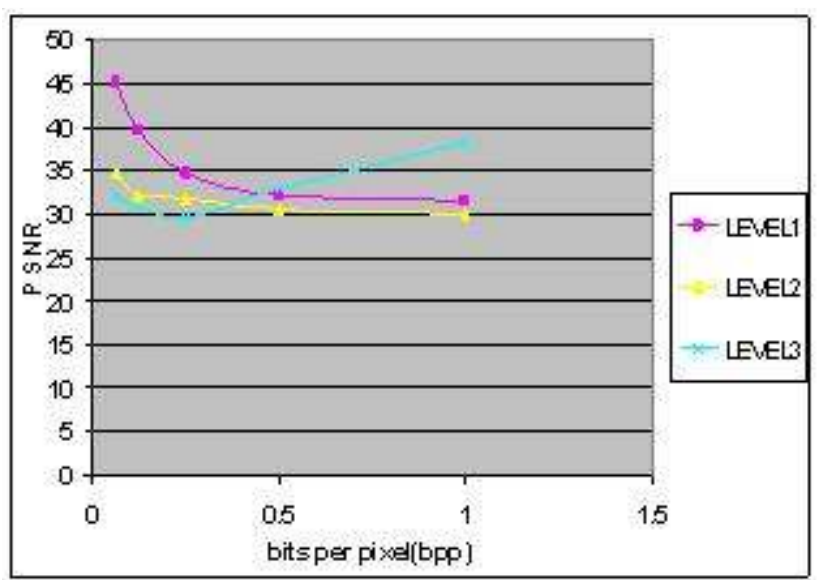

Figure 19 PSNR response db6

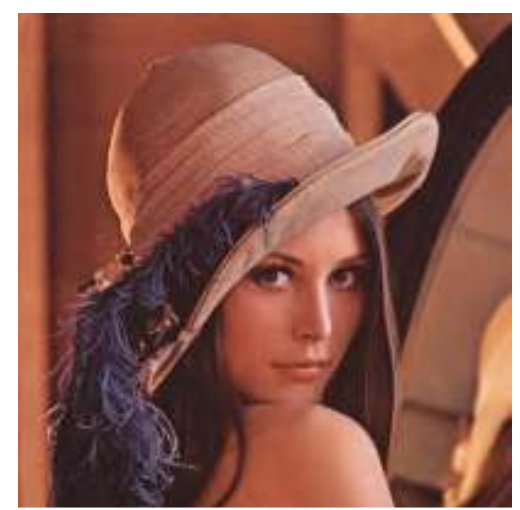

Figure 20:Reconstructed image for db6

\section{- $\quad$ db9}

Table 1.9 shows PSNR values for wavelet db9 for different level of decomposition. Bits per Pixel are kept at 1bpp for our analysis and level of decomposition is kept fixed to 3. Figure 21 shows the PSNR response plotted for the values given in table 1.9. Figure 22 shows the reconstructed image for coif4 wavelet.

Table 1.9 PSNR for different level of wavelet decomposition for $\mathbf{d b} 9$

\begin{tabular}{|l|l|l|l|l|l|}
\hline bpp & 0.0625 & 0.125 & 0.25 & 0.5 & 1 \\
\hline Level & \multicolumn{5}{|c|}{ PSNR } \\
\hline 1 & 45.11 & 39.85 & 34.70 & 32.11 & 31.68 \\
\hline 2 & 34.86 & 32.38 & 31.9 & 30.64 & 29.98 \\
\hline 3 & 32.06 & 30.73 & 29.56 & 32.83 & 38.17 \\
\hline
\end{tabular}




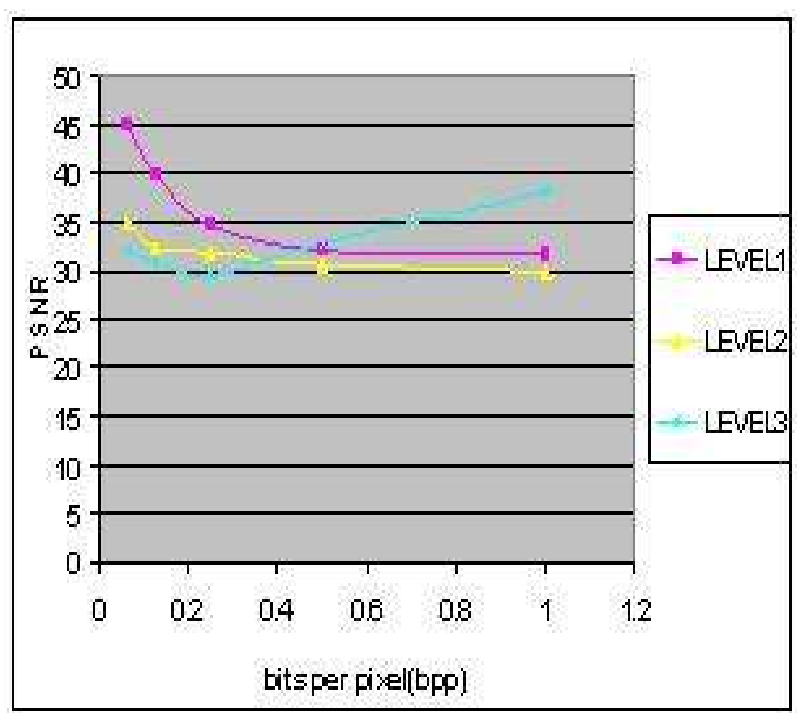

Figure 21: PSNR response of $\mathrm{db9}$

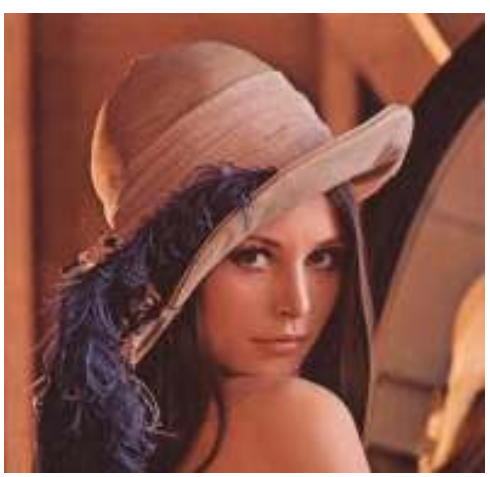

Figure 22: Reconstructed image for $\mathbf{d b 9}$

\section{- HAAR}

Table 2.0 shows PSNR values for wavelet HAAR for different level of decomposition. Bits per Pixel are kept at 1bpp for our analysis and level of decomposition is kept fixed to 3 . Figure 23 shows the PSNR response plotted for the values given in table 2.0. Figure 24 shows the reconstructed image for coif4 wavelet.

Table 2.0: PSNR for different level of wavelet decomposition for HAAR

\begin{tabular}{|l|l|l|l|l|l|}
\hline bpp & 0.0625 & 0.125 & 0.25 & 0.5 & 1 \\
\hline Level & \multicolumn{5}{|c|}{ PSNR } \\
\hline 1 & 44.40 & 39.19 & 34.29 & 31.74 & 31.34 \\
\hline 2 & 34.27 & 31.80 & 31.39 & 30.25 & 29.97 \\
\hline 3 & 31.47 & 30.27 & 29.72 & 32.58 & 36.92 \\
\hline
\end{tabular}

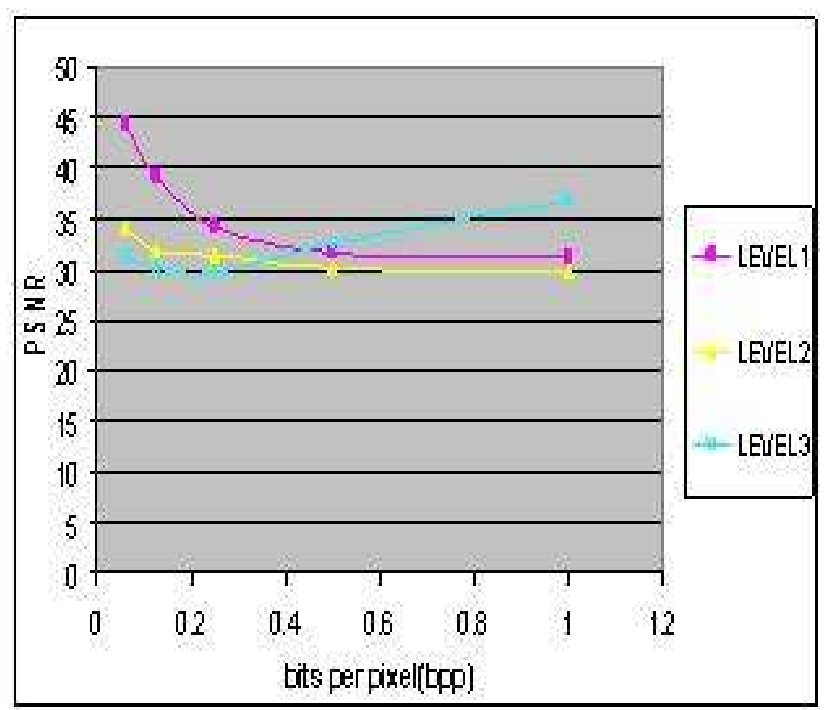

Figure 23:PSNR response of HAAR

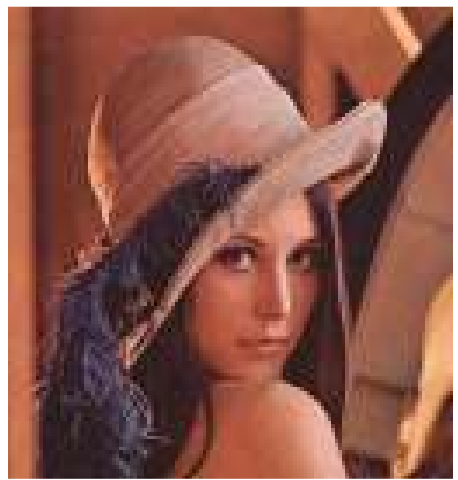

Figure 24: Reconstructed image for HAAR

\section{- SYM3}

Table 2.1 shows PSNR values for wavelet SYM3 for different level of decomposition. Bits per Pixel are kept at $1 \mathrm{bpp}$ for our analysis and level of decomposition is kept fixed to 3. Figure 25 shows the PSNR response plotted for the values given in table 1.6. Figure 26 shows the reconstructed image for coif 4 wavelet.

Table 2.1 PSNR for different level of wavelet decomposition for SYM3

\begin{tabular}{|l|l|l|l|l|l|}
\hline bpp & 0.0625 & 0.125 & 0.25 & 0.5 & 1 \\
\hline Level & \multicolumn{5}{|c|}{ PSNR } \\
\hline 1 & 44.99 & 39.56 & 34.63 & 32.07 & 31.64 \\
\hline 2 & 34.61 & 32.16 & 31.73 & 30.47 & 30.06 \\
\hline 3 & 32.05 & 30.66 & 29.8 & 32.91 & 38.20 \\
\hline
\end{tabular}




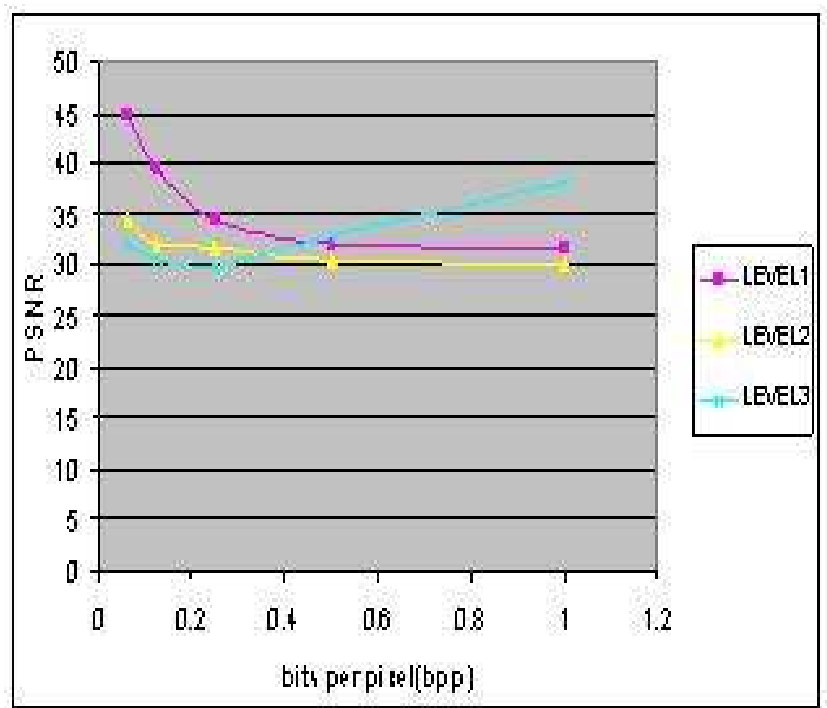

Figure 25:PSNR response of SYM3

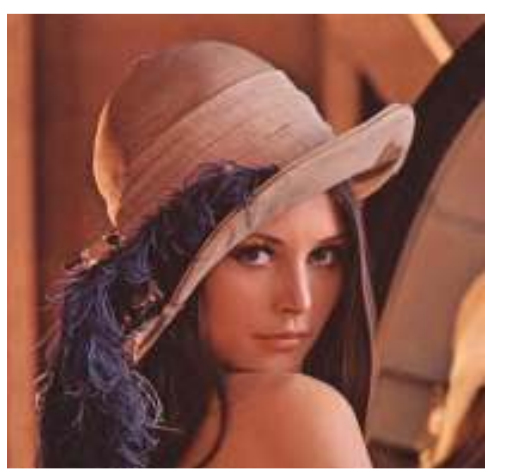

Figure 26:Reconstructed image for SYM3

\section{- $\quad$ SYM7}

Table 2.2 shows PSNR values for wavelet SYM7 for different level of decomposition. Bits per Pixel are kept at 1bpp for our analysis and level of decomposition is kept fixed to 3 . Figure 27 shows the PSNR response plotted for the values given in table 2.2. Figure 28 shows the reconstructed image for coif4 wavelet.

Table 2.2 PSNR for different level of wavelet decomposition for SYM7

\begin{tabular}{|l|l|l|l|l|l|}
\hline bpp & 0.0625 & 0.125 & 0.25 & 0.5 & 1 \\
\hline Level & \multicolumn{5}{|c|}{ PSNR } \\
\hline 1 & 44.849 & 39.469 & 34.615 & 32.023 & 31.604 \\
\hline 2 & 34.658 & 32.238 & 31.799 & 30.497 & 30.022 \\
\hline 3 & 32.069 & 30.659 & 29.754 & 32.950 & 38.402 \\
\hline
\end{tabular}

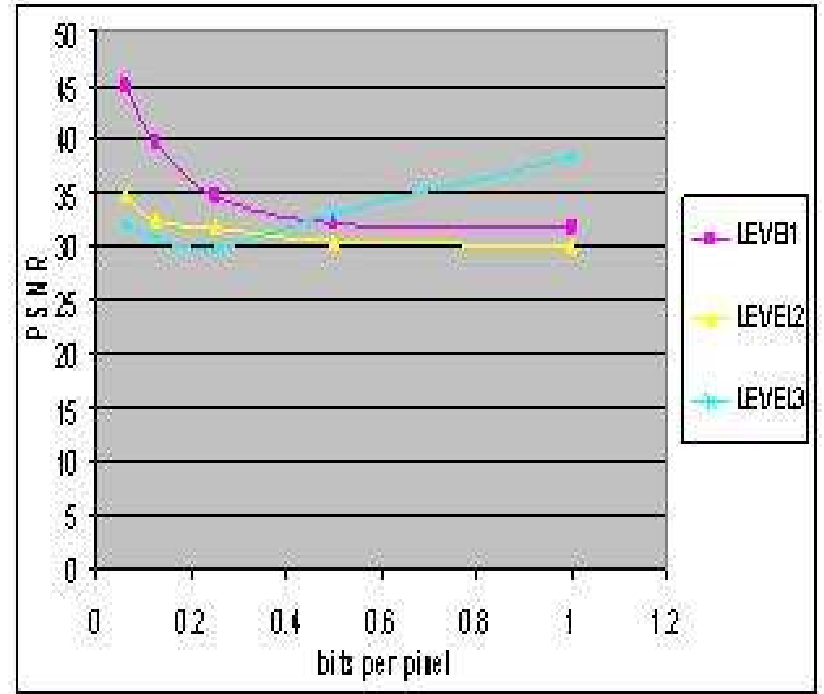

Figure 27: PSNR response of SYM7

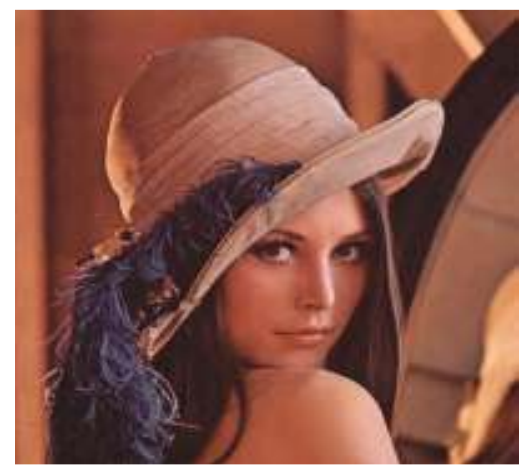

Figure 28: Reconstructed Image for SYM7

\section{CONCLUSION AND FUTURE WORKS}

Compressing color images efficiently are one of the main problems in multimedia applications. So we have tested the efficiency of color image compression using SPIHT algorithm. The SPIHT algorithm is applied for luminance (Y) and chrominance $(\mathrm{Cb}, \mathrm{Cr})$ part of $\mathrm{RGB}$ to $\mathrm{YCbCr}$ transformed image. Reconstructed image is verified using human vision and PSNR. Huffman and arithmetic coding can be added to increase the compression. We can test the channel behavior by sending compressed image between two computer and check the reconstructed image.

\section{REFERENCES}

[1] Said A, Pearlman WA. "A new fast and efficient image codec based on set partitioning in hierarchical trees". IEEE Transactions on Circuits and Systems for Video Technology 1996;6:243-50.

[2] J M Shapiro, "Embedded image coding using Zero trees of wavelet coefficients", IEEE Trans. Signal Processing, Vol 41, pp 3445-3462, Dec 1993. 
[3] Rafael C. Gonzalez and Richard E. Woods. Digital Image Processing. Pearson Education, Englewood Cliffs,2002 .

[4] K.Sayood, "Introduction to Data Compression", 2nd edition, Academic Press, Morgan Kaufman Publishers, 2000.

[5] G.Sadashivappa, K.V.S.AnandaBabu,"Performance analysis of Image Coding of Wavelets" IJCSNS International Journal of Computer Science and Network Security, Oct 2008.

[6] G.Sadashivappa, K.V.S.AnandaBabu, "Wavelet Filters for Image Compression, an analytical study" ICGST-GVIP Journal, Volume 9, Issue 5, September 2009.

[7] K.P.Soman,K.I.Ramachandran "Insight into Wavelets from theory to practice". Prentice-Hall of India Private Limited.

[8] EZW present at Website: http://pagespersoorangefr/polyvalens/Clemens/ezw/ ezw $\cdot$ html

[9] ANTONINI, M., BARLAUD, M., MATHIEU, P., and DAUBECHIES, I.: 'Image coding using wavelet transform', IEEE Trans. Image Process., 1992,1,(2),pp.205-220.

\section{AUTHORS PROFILE}

G.Sadashivappa, received the BE degree in Electronics Engineering from Bangalore University in 1984 and M.Tech degree in Industrial Electronics from NIT-k, Mangalore University in1991. Since 1992, is working in R.V.College of Engineering. He is pursuing his Doctoral program at VTU Belgaum. His research interests are Image and Video Coding, Signal Processing and fiber optic networks.

Mahesh Jayakar, received the BE degree in Electronics and Communication Engineering from NMIT, Bangalore in 2008. Currently he is working in R.V.College of Engineering.

Dr. K.V.S Ananda Babu, graduated from IIT Madras in 1969, obtained M.Tech from IIT Delhi in1983. He obtained doctorate from Osmania University, Osmania in 2000. Currently working as principal CMRIT, Bangalore. 